\title{
$\mathrm{Ni}_{0.4} \mathrm{Co}_{0.2} \mathrm{Zn}_{0.4} \mathrm{Fe}_{2} \mathrm{O}_{4} / \mathrm{BaTiO}_{3}$ 纳米纤维双层吸波涂层的 微波吸收特性研究
}

\author{
李佳乐 ${ }^{1}$, 向 军 ${ }^{1}$, 叶 芹 ${ }^{1}$, 刘 敏 ${ }^{1}$, 沈湘黔 ${ }^{2}$ \\ (1. 江苏科技大学 数理学院, 镇江 212003; 2. 江苏大学 材料科学与工程学院, 镇江 212013)
}

摘 要: 采用静电纺丝法制备了平均直径分别为 $180 \mathrm{~nm}$ 和 $220 \mathrm{~nm}$ 的 $\mathrm{BaTiO}_{3}(\mathrm{BTO})$ 和 $\mathrm{Ni}_{0.4} \mathrm{Co}_{0.2} \mathrm{Zn}_{0.4} \mathrm{Fe}_{2} \mathrm{O}_{4}(\mathrm{NCZFO})$ 纳米纤维, 使用 X 射线衍射(XRD)、场发射扫描电镜(FESEM)和矢量网络分析仪(VNA)对纤维的物相结构、表面形 貌和微波电磁参数进行了表征, 并根据传输线理论分析评估了以 BTO 和 NCZFO 纳米纤维为吸收剂的硅橡胶基单 层和双层结构吸波涂层在 $2 \sim 18 \mathrm{GHz}$ 范围内的微波吸收性能。结果显示, 由于 BTO 纳米纤维的介电损耗与 NCZFO 纳米纤维的磁损耗的有机结合和阻抗匹配特性的改善, 以 NCZFO 纳米纤维/硅橡胶复合体(S1)为匹配层、BTO 纳米纤维 /硅橡胶复合体 $(\mathrm{S} 2)$ 为吸收层的双层吸波涂层比相应单层吸波涂层表现出更为优异的吸收性能。通过调节匹配层与 吸收层的厚度, 在 4.9 18 GHz范围内反射损耗可达 $-20 \mathrm{~dB}$ 以下; 当吸收层和匹配层的厚度分别为 $2.3 \mathrm{~mm}$ 和 $0.5 \mathrm{~mm}$ 时, 最小反射损耗位于 $9.5 \mathrm{GHz}$ 达- $87.8 \mathrm{~dB}$, 低于-20 dB 的吸收带宽为 $5 \mathrm{GHz}$ 。优化设计的 $\mathrm{NCZFO} / \mathrm{BTO}$ 纳米纤维 双层吸波涂层有望发展成为一种新型的宽频带强吸收吸波材料。

关 键 词: Ni-Co- $\mathrm{Zn}$ 铁氧体纳米纤维; $\mathrm{BaTiO}_{3}$ 纳米纤维; 静电纺丝; 双层吸波涂层; 微波吸收特性 中图分类号: TM25; TQ343 文献标识码: A

\section{Microwave Absorption Properties of Double-layer Absorbing Coatings Based on $\mathrm{Ni}_{0.4} \mathrm{Co}_{0.2} \mathrm{Zn}_{0.4} \mathrm{Fe}_{2} \mathrm{O}_{4}$ and $\mathrm{BaTiO}_{3}$ Nanofibers}

\author{
LI Jia-Le ${ }^{1,2}$, XIANG Jun ${ }^{1}$, YE Qin ${ }^{1}$, LIU Min ${ }^{1}$, SHEN Xiang-Qian ${ }^{2}$
}

(1. School of Mathematics and Physics, Jiangsu University of Science and Technology, Zhenjiang 212003, China; 2. School of Material Science and Engineering, Jiangsu University, Zhenjiang 212013, China)

\begin{abstract}
BaTiO}_{3}$ (BTO) nanofibers (NFs) and $\mathrm{Ni}_{0.4} \mathrm{Co}_{0.4} \mathrm{Zn}_{0.4} \mathrm{Fe}_{2} \mathrm{O}_{4}$ (NCZFO) NFs with average diameters of about $180 \mathrm{~nm}$ and $220 \mathrm{~nm}$, respectively, were prepared by electrospinning method. Their phase structures, surface morphologies and electromagnetic parameters were characterized by X-ray diffraction (XRD), field mission scanning electron microscope (FESEM) and vector network analyzer. Microwave absorption properties of both singleand double-layer silicone rubber based absorbing coatings with either 70wt\% BTO NFs or NCZFO NFs as microwave absorbents were evaluated according to the transmission line theory in the frequency range of 2-18 GHz. The results show that the double-layer absorbing coatings with the NCZFO NFs/silicone rubber composite (S1) as
\end{abstract}

收稿日期: 2014-10-11; 收到修改稿日期: 2014-12-01

基金项目：国家自然科学基金(51274106); 博士后科学基金(2013M540418); 江苏省博士后科研资助计划(1301055B); 江苏 省普通高校研究生科研创新计划(KYLX-1084)

National Natural Science Foundation of China (51274106); China Postdoctoral Science Foundation (2013M540418); Jiangsu Planned Projects for Postdoctoral Research Funds (1301055B); Postgraduate Research and Innovation Project of University in Jiangsu Province (KYLX-1084)

作者简介: 李佳乐(1990-), 男, 硕士研究生. E-mail: 1jljust@163.com

通讯作者：向 军，副教授. E-mail: jxiang@just.edu.cn 
matching layer and the BTO NFs/silicone rubber composite (S2) as absorbing layer display superior microwave absorbing performance compared to the single-layer ones due to the proper combination of magnetic loss of NCZFO NFs and dielectric loss of BTO NFs, and the improved impedance matching characteristics. The double-layer absorbing coatings have a reflection loss (RL) of less than $-20 \mathrm{~dB}$ over the frequency range of 4.9-18 GHz through appropriately adjusting the thicknesses of the absorbing layer and matching layer. When the thicknesses of the absorbing layer and the matching layer are 2.3 and $0.5 \mathrm{~mm}$, respectively, the minimum RL reaches $-87.8 \mathrm{~dB}$ at $9.5 \mathrm{GHz}$ and the absorption bandwidth with the RL values below $-20 \mathrm{~dB}$ is up to $5 \mathrm{GHz}$. The optimal NCZFO NFs/BTO NFs double-layer absorbing coatings can become a novel microwave absorption material with strong-absorption and broad-band.

Key words: Ni-Co-Zn ferrite nanofiber; $\mathrm{BaTiO}_{3}$ nanofiber; electrospinning; double-layer absorber; microwave absorption properties

近年来, 随着 $\mathrm{GHz}$ 频段电磁波在电子通讯领域 的广泛应用, 如移动电话、无线局域网、雷达系统 等, 带来的电磁辐射、干扰与污染也越发严重; 另一 方面, 提高现代战争中武器装备的生存和突防能力 要求尽可能降低雷达反射截面以实现其电磁隐身。 于是为了解决电磁干扰以及军事装备隐身问题, 高 性能电磁波吸收材料的研制与开发一直是相关领域 的热点课题 ${ }^{[1-5]}$ 。微波吸收材料的吸波性能主要由其 相对复磁导率和复介电常数所决定 ${ }^{[6]}$ 。性能优异的 微波吸收材料必须同时满足两个条件: 一是阻抗匹 配良好, 入射电磁波能有效进入吸波体内部而不被 反射; 二是衰减能力强, 进入到材料内部的电磁波 能被迅速地衰减和吸收 ${ }^{[7-8]}$ 。

吸波材料可分为单层和多层结构, 单层吸波材 料通常难以同时达到低厚度、宽频带、强吸收的效 果，而双层及多层吸波材料由于其更易实现与自由 空间的阻抗匹配, 获得好的微波吸收性能, 则近年 来引起了研究者的广泛关注 ${ }^{[9-13]}$ 。 Liu 等 ${ }^{[9]}$ 设计了以 $\mathrm{Co}_{2} \mathrm{FeO}_{4}$ 和羟基铁 $(\mathrm{CI})$ 粉为吸收剂的双层吸波涂层, 最小反射损耗达到 $-38.2 \mathrm{~dB}$, 低于 $-10 \mathrm{~dB}$ 的吸收带 宽为 $9.4 \mathrm{GHz}$; $\mathrm{Qing}$ 等 $^{[10]}$ 模拟计算了以树脂/ $(50 \mathrm{wt} \%) \mathrm{BaTiO}_{3}$ 复合材料为匹配层和树脂 $/(60 \mathrm{wt} \%)$ $\mathrm{BaTiO}_{3} /(20 \mathrm{wt} \%) \mathrm{CI}$ 复合材料为吸收层的双层吸波材 料, 其最小反射损耗达 $-59 \mathrm{~dB}$, 小于 $-10 \mathrm{~dB}$ 的吸收 频带为 10.8 14.8 GHz。这些双层吸波材料均比相应 单层吸波材料表现出更强的微波吸收性能。

尖晶石结构铁氧体由于其磁损耗大、吸收能力 强被广泛应用于电磁波吸收领域, 但相应单层吸波涂 层通常大多存在密度高、匹配厚度大等不足之处 ${ }^{[14-15]}$ 。 钙钛矿结构 $\mathrm{BaTiO}_{3}(\mathrm{BTO})$ 具有良好的介电和铁电性 能, 近来发现一些 BTO 纳米结构呈现出较强的电磁 吸收能力 ${ }^{[16-20]}$ 。然而由于此类材料仅有介电损耗,
造成在单独使用时吸波涂层的阻抗匹配特性较差, 存在吸收频带窄等缺点。本研究采用静电纺丝技术 结合后期热处理制备了 BTO 和 $\mathrm{Ni}_{0.4} \mathrm{Co}_{0.2} \mathrm{Zn}_{0.4} \mathrm{Fe}_{2} \mathrm{O}_{4}$ (NCZFO) 纳米纤维微波吸收剂, 分析评估了电磁 特性和硅橡胶基单层与双层吸波涂层的微波吸收 性能。

\section{1 实验方法}

\section{1 药品}

乙酸镍、乙酸锌和钛酸四丁酯，均为分析纯，购 于阿拉丁试剂(上海)有限公司; 硝酸钴、硝酸铁、乙 酸钡、无水乙醇和乙酸, 均为分析纯, 购于国药集团 化学试剂有限公司; 聚乙烯吡咯烷酮(PVP), 平均 分子量 1300000 , 购于西格玛奥德里奇(上海)贸易 有限公司。

\subsection{NCZFO 纳米纤维的制备}

先将 $1.2 \mathrm{~g} \mathrm{PVP}$ 溶于 $10.133 \mathrm{~g}$ 无水乙醇和 $5.067 \mathrm{~g}$ 去离子水所组成的混合溶剂中, 在室温下磁力搅拌 约 $1 \mathrm{~h}$ 使 PVP 完全溶解。然后根据目标产物中各金 属元素的化学计量比称取 $0.340 \mathrm{~g}$ 乙酸镍、 $0.199 \mathrm{~g}$ 硝酸钴、 $0.300 \mathrm{~g}$ 乙酸锌和 $2.761 \mathrm{~g}$ 硝酸铁加入到上 述 PVP 溶液中, 继续磁力搅拌约 $12 \mathrm{~h}$ 后制得均匀透 明的纺丝溶液, 其中金属盐与 PVP 的质量分数分别 为 $18 \%$ 和 $6 \%$ 。将配制好的溶液通过自制的静电纺 丝装置, 在溶液推进速度 $0.5 \mathrm{~mL} / \mathrm{h}$ 、电压 $15 \mathrm{kV}$ 和 接收距离 $15 \mathrm{~cm}$ 的条件下电纺制成 PVP/NCZFO 前 驱体纤维。收集到的前驱体纤维经充分干燥后在 $850{ }^{\circ} \mathrm{C}$ 焙烧 $2 \mathrm{~h}$ 制得 $\mathrm{NCZFO}$ 纳米纤维, 升温速率为 $2{ }^{\circ} \mathrm{C} / \mathrm{min}$ 。

\subsection{BTO 纳米纤维的制备}

称取 1.029 乙酸钡加入到 $12.600 \mathrm{~g}$ 二甲基甲酰 
胺(DMF)和 $3.000 \mathrm{~g}$ 乙酸组成的混合溶剂中, 在室温 下磁力搅拌约 $0.5 \mathrm{~h}$ 至乙酸钡完全溶解后再向其中 逐滴滴加 $1.371 \mathrm{~g}$ 钛酸四丁酯 $(\mathrm{Ba}$ 与 $\mathrm{Ti}$ 物质的量之 比为 $1: 1)$, 最后再加入 $2.000 \mathrm{~g} \mathrm{PVP}$, 经磁力搅拌约 $4 \mathrm{~h}$ 配制成纺丝溶液, 其中 PVP 和金属盐的质量百 分含量分别为 $10 \%$ 和 $12 \%$ 。将所得溶液移入到自制 静电纺丝装置中，在溶液推进速率 $0.5 \mathrm{~mL} / \mathrm{h}$ 、电压 $15 \mathrm{kV}$ 、接收距离 $20 \mathrm{~cm}$ 的条件下电纺制成 PVP/BTO 前驱体纤维, 经适当干燥后以 $2{ }^{\circ} \mathrm{C} / \mathrm{min}$ 的速率从室 温加热至 $850{ }^{\circ} \mathrm{C}$ 保温 $2 \mathrm{~h}$ 得到 $\mathrm{BTO}$ 纳米纤维。

\section{4 样品表征}

采用日本电子的 JSM-7001F 型场发射扫描电 子显微镜(FESEM)观察纤维的微观形貌。利用日本 岛津的 XRD-6000 型 X 射线衍射仪(XRD, $\mathrm{Cu}$ 靶, $\mathrm{K} \alpha$ 射线, $\lambda=0.15406 \mathrm{~nm}$, 管电流 $30 \mathrm{~mA}$, 管电压 $40 \mathrm{kV}$, $2 \theta$ 角范围为 $20^{\circ} \sim 70^{\circ}$, 扫描速度 $5^{\circ} / \mathrm{min}$, 步长 $0.02^{\circ}$ ) 分析样品的物相结构。使用同轴线传输/反射法测 量所得纤维材料的电磁参数, 以硅橡胶 (Dow Corning 184)为基质, 将 $\mathrm{BTO}$ 纳米纤维和 NCZFO 纳米纤维各自以质量比 7:3 的比例与硅橡胶及固化 剂均匀混合, 然后将混合物装填到钢制模具中经 $120{ }^{\circ} \mathrm{C}$ 固化 $1 \mathrm{~h}$ 制成外径 $7.00 \mathrm{~mm}$ 、内径 $3.04 \mathrm{~mm}$ 、 厚约 $2 \mathrm{~mm}$ 的环状试样, 在美国安捷伦 E5071C 型 矢量网络分析仪上测量它们在 2 18 GHz 频率范围 内的复介电常数和复磁导率, 根据传输线理论模 拟计算和分析相应单层和双层结构吸波涂层的微 波吸收性能。

\section{2 结果与讨论}

\section{1 纤维的物相结构与形貌}

图 1 是 $\mathrm{NCZFO}$ 和 BTO 纳米纤维的 XRD 图谱, 由图可见, NCZFO 和 BTO 纳米纤维所出现的衍射 峰可各自按立方尖晶石结构(PDF \# 08-0234)和四方 钙钛矿结构(PDF \# 05-0626)进行指标化, 均无其他 杂相存在, 显示它们均为单相结构。此外可观察到, 所有特征衍射峰都比较尖锐, 说明所制得的纤维晶 化程度较高, 晶格发育较为完整。基于 NCZFO 和 BTO 主峰(311)和(110)的衍射数据, 利用 Scherrer 公 式估算得到 NCZFO 与 BTO 纳米纤维的平均晶粒尺 寸分别约为 53 和 $34 \mathrm{~nm}$ 。

图 2 为 NCZFO 与 BTO 纳米纤维的 FESEM 照 片。从图中可见, NCZFO 和 BTO 纳米纤维粗细较为 均匀, 但表面比较粗糙, 平均直径分别约为 220 和 $180 \mathrm{~nm}$ 。对于 NCZFO 纳米纤维(图 2(a)), 铁氧体颗

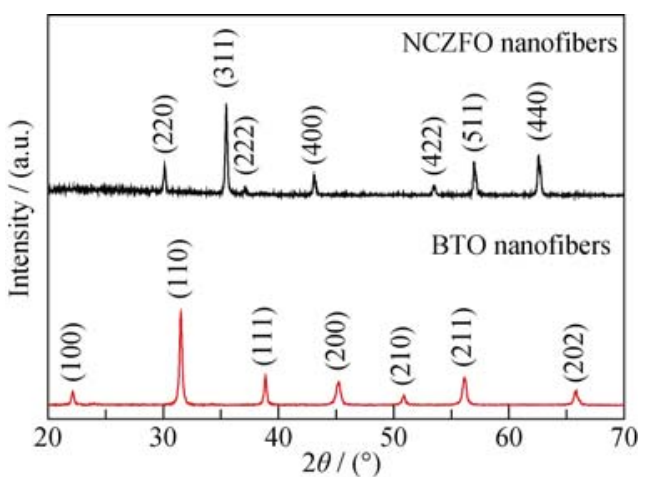

图 $1 \mathrm{NCZFO}$ 与 $\mathrm{BTO}$ 纳米纤维的 XRD 图谱

Fig. 1 XRD patterns of as-prepared NCZFO and BTO nanofibers
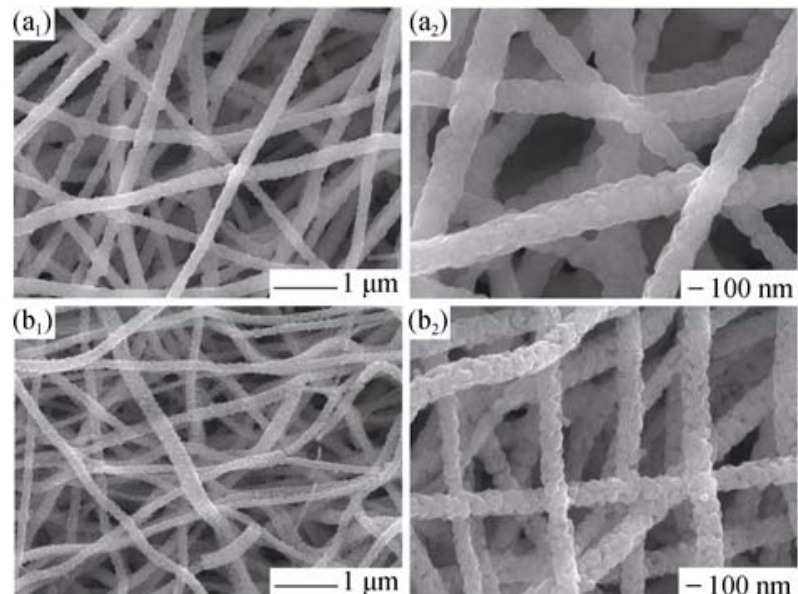

图 2 NCZFO 纳米纤维 $\left(a_{1}, a_{2}\right)$ 和 BTO 纳米纤维 $\left(b_{1}, b_{2}\right)$ 的 FESEM 照片

Fig. 2 FESEM images of $\operatorname{NCZFO}\left(a_{1}, a_{2}\right)$ and BTO $\left(b_{1}, b_{2}\right)$ nanofibers

粒间接触紧密，整个纤维显得比较致密; 而 BTO 纳 米纤维(图 2(b)), 其微观结构则显得有些松散。这可 能是热处理过程中形成的 BTO 粒子相对较小, 无法 完全弥合前驱体纤维中有机物和金属盐分解形成的 大量孔洞，导致所得 BTO 纳米纤维呈现出疏松多孔 结构。此外, 由于在高温焙烧过程中晶粒的融合生 长, 从图 2 还可观察到部分纤维出现了相互交联的 现象。

\section{2 电磁特性}

复介电常数和复磁导率的实部和虚部分别代表 对材料内传播的电磁波能量的储存和损耗能力 ${ }^{[21]}$ 。

图 3 是 NCZFO 纳米纤维/硅橡胶复合物(记为 $\mathrm{S} 1$ )的 复介电常数、复磁导率和损耗角正切随频率的变化 曲线。由图 3(a)可见, $\mathrm{S} 1$ 的复介电常数实部 $\left(\varepsilon^{\prime}\right)$ 与虚 部 $\left(\varepsilon^{\prime \prime}\right)$ 在 $2 \sim 18 \mathrm{GHz}$ 内几乎保持不变, 其值分别在 5.02 和 0.15 左右。由于整个介电谱没有出现明显的 松弛和共振行为，偶极子的取向极化可能是该频率 范围内的主要极化机制 ${ }^{[15]}$ 。从图 3(b)可观察到, 复 

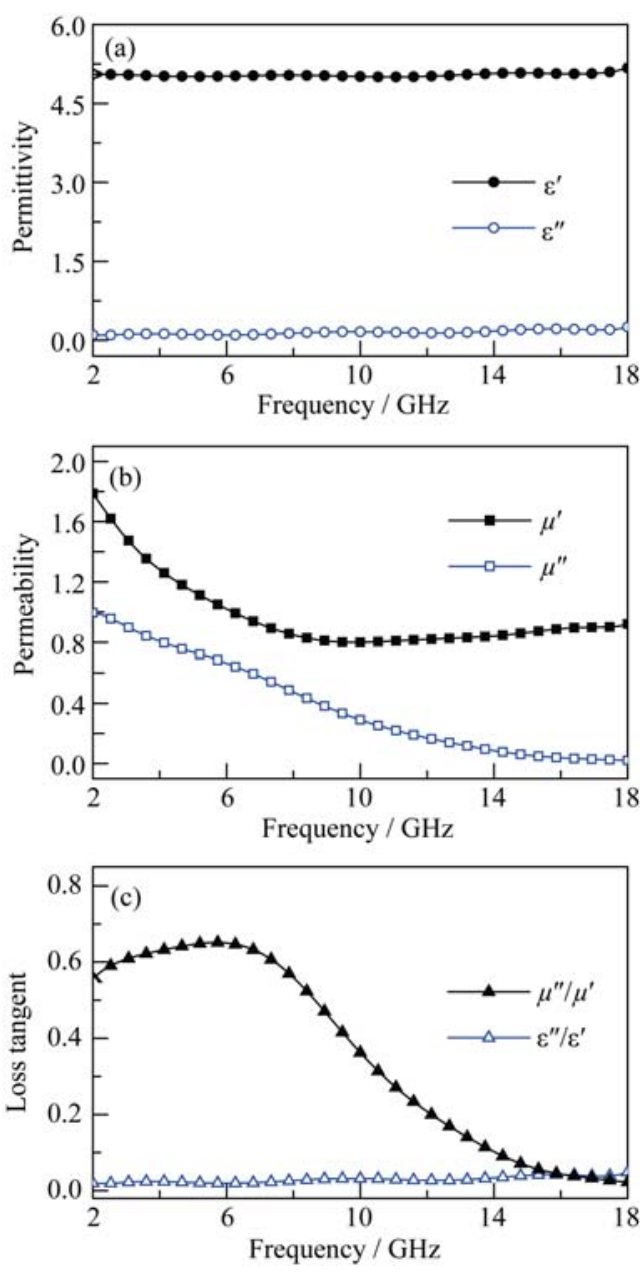

图 3 NCZFO 纳米纤维/硅橡胶复合物(S1)的(a)复介电常数、 (b)复磁导率和(c)损耗角正切随频率的变化

Fig. 3 Frequency dependences of (a) complex permittivity, (b) complex permeability and (c) loss tangent of S1

磁导率实部 $\left(\mu^{\prime}\right)$ 在 2 9 $\mathrm{GHz}$ 范围内从 1.79 较快减小 到 0.81 , 之后随频率升高平缓增大至 0.92 ; 而复磁 导率虚部 $\left(\mu^{\prime \prime}\right)$ 在整个测量频率范围内呈单调下降趋 势。损耗角正切大小反映了材料对电磁波的衰减能 力, 从图 3(c) 可以看出, $\mathrm{S} 1$ 的介电损耗角正切 $\left(\varepsilon^{\prime \prime} / \varepsilon^{\prime}\right)$ 接 近于 0 , 而磁损耗角正切 $\left(\mu^{\prime \prime} / \mu^{\prime}\right)$ 几乎在整个 $2 \sim 18 \mathrm{GHz}$ 范围内都大于相应的介电损耗角正切, 其最大值在 $6 \mathrm{GHz}$ 附近达到 0.65 , 说明 NCZFO 纳米纤维对电磁 波能量的衰减主要来自于磁损耗, 同时也预示着该 纤维材料对中低频电磁波具有一定的吸收能力。一 般而言, 磁性材料在 $\mathrm{GHz}$ 范围内的磁损耗主要归于 自然共振、浴流损耗和交换共振 $[5,22]$ 。铁氧体材料 的电阻率较高, 浴流损耗可以忽略。尖晶石铁氧体 的自然共振频率虽然一般都在数百 $\mathrm{MHz}$ 以下 ${ }^{[23]}$, 但对于其纳米结构体系，由于小尺寸效应显著提高 了表面各向异性, 使自然共振频率可达 $\mathrm{GHz}$ 范围, 从而能够在微波频段表现出较强的磁损耗能力。因
此, NCZFO 铁氧体纳米纤维在微波频段的磁频散及 磁损耗应主要由自然共振引起 ${ }^{[14]}$ 。此外, 当磁性材 料尺度达到纳米级时, 体系中磁性颗粒间交换相互 作用将显著增强，交换能增大，交换共振对磁损耗 可能也会产生一定的贡献 ${ }^{[14,22]}$ 。

图 4 是 BTO 纳米纤维/硅橡胶复合体(记为 S2) 的复介电常数、复磁导率和损耗角正切随频率的变 化曲线。由图 4(a)可见, $\varepsilon^{\prime}$ 在 2 15.6 GHz 频率范围内 基本都在 16.2 左右, 之后随频率升高快速下降到 12.6。由于电子和离子极化分别出现在紫外区和红 外区 ${ }^{[22]}$, BTO 在微波频段的介电常数及极化程度应 主要取决于其本身固有偶极子的取向极化 ${ }^{[20,24]}$ 。但 随微波频率升高到一定值后, BTO 固有电偶极矩的 取向重排将跟不上外电场的变化而失去响应，使 $\varepsilon^{\prime}$ 在高频区出现下降。此外, 由于 BTO 纳米纤维是由 BTO 晶粒聚集构成, 晶粒界面会聚集电荷形成偶极 矩, 因此界面极化可能对其介电常数也具有一定贡
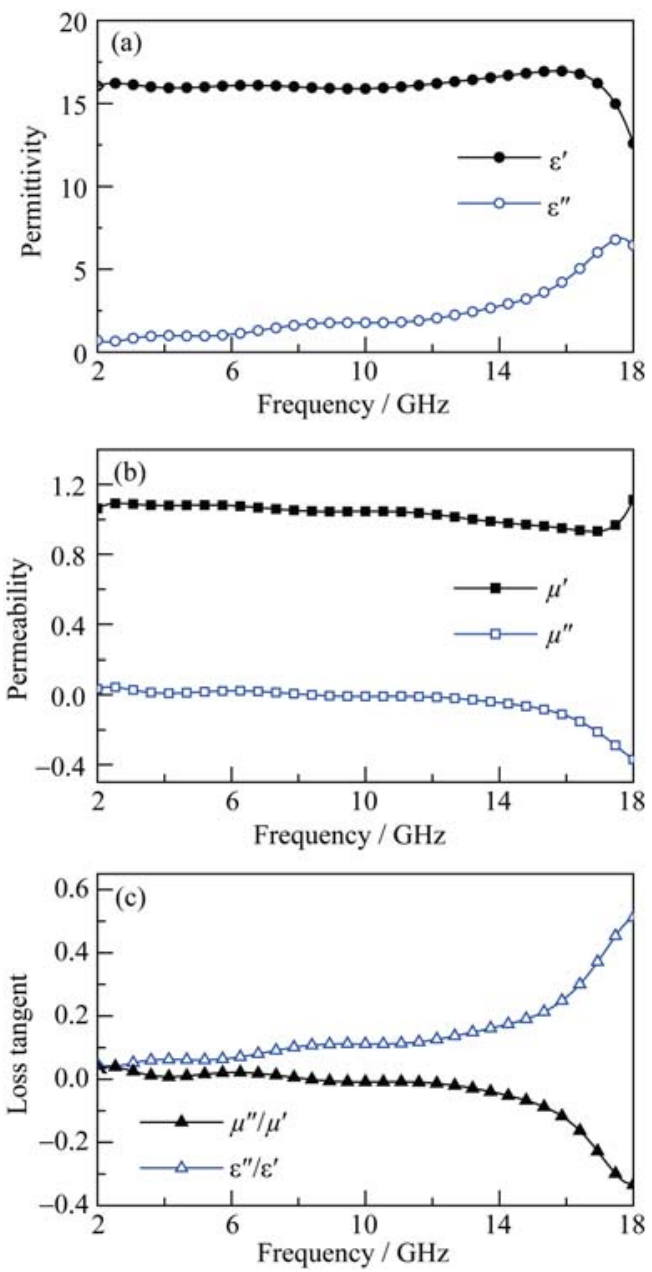

图 4 BTO 纳米纤维/硅橡胶复合体(S2)的(a)复介电常数、(b) 复磁导率和 $(\mathrm{c})$ 损耗角正切随频率的变化

Fig. 4 Frequency dependences of (a) complex permittivity, (b) complex permeability and (c) loss tangent of S2 
献。复介电常数虚部值在 $2 \sim 18 \mathrm{GHz}$ 内总体呈逐渐 增大趋势, 从 0.7 增加到约 6.6 , 但在中低频范围内 (2 12 GHz)其值及增幅都相对比较小，相应的介电 损耗角正切曲线[图 4(c)]也表现出相同的变化行为。 当频率相对较低时, 介电损耗主要由漏导所决定, 相应损耗基本不依赖于频率; 而当频率升高到高频 段时，介电损耗机制则主要是弛豫极化损耗和电导 损耗 ${ }^{[10]}$ 。于是 BTO 纳米纤维的介电损耗出现在中 低频段变化较小，高频范围大幅增加的现象。从图 4(b)可观察到, S2 的 $\mu^{\prime}$ 和 $\mu^{\prime \prime}$ 几乎在整个测量频率范 围内都各自接近于 1 和 0 , 没有明显的磁损耗, 这是 由于 BTO 为非磁性介质。不过值得注意的是, $\mu^{\prime \prime}$ 和 磁损耗角正切在高频段随频率增大而下降变成了负 值, 出现负磁导率现象。该现象在多孔碳 ${ }^{[25]} 、 \mathrm{Fe}_{3} \mathrm{O}_{4} /$ 碳微管纳米复合物 ${ }^{[26]}$ 和 $\mathrm{Fe} / \mathrm{Co} / \mathrm{Ni}$-碳复合纳米纤维 ${ }^{[5]}$ 等体系中也有所报道。根据 Maxwell 电磁理论, 处 于交变磁场中的载流子在感生电场作用下形成浴电 流，并产生一个相反的感应磁场，结果使部分电场 能转换成磁场并以磁场能的形式从材料内辐射出来, 导致磁导率虚部下降成为负值。此外, 从图 4(c)可 见，在整个 2 18 GHz 频率范围内, S2 的介电损耗角 正切均高于相应的磁损耗角正切, 说明其微波吸收 机制主要是介电损耗。

\section{3 吸波性能分析}

根据传输线理论，单层吸波涂层在电磁波垂直 入射时的反射损耗 $(R L)$ 可通过下列公式进行数值模 拟计算 ${ }^{[10,27]}$ :

$$
\begin{gathered}
R L(\mathrm{~dB})=20 \lg \left|Z_{\text {in }}-Z_{0} / Z_{\text {in }}+Z_{0}\right| \\
Z_{\text {in }}=Z_{0} \sqrt{\mu_{\mathrm{r}} / \varepsilon_{\mathrm{r}}} \tanh \left[j(2 \pi f d / c) \sqrt{\mu_{\mathrm{r}} \varepsilon_{\mathrm{r}}}\right]
\end{gathered}
$$

式中, $Z_{0}$ 为自由空间的特征阻抗; $Z_{\text {in }}$ 为吸波涂层的 输入阻抗; $\varepsilon_{\mathrm{r}}$ 和 $\mu_{\mathrm{r}}$ 是吸波涂层的相对复介电常数和 复磁导率; $d$ 为吸波涂层的厚度; $f$ 是入射电磁波的 频率; $\mathrm{c}$ 为光速。

图 5 是根据所测电磁参数计算得到的不同厚度 $\mathrm{S} 1$ 和 $\mathrm{S} 2$ 单层吸波涂层在 2 18 GHz 内的反射损耗曲 线。从图 5(a)可以看出, $\mathrm{S} 1$ 单层在所考察的厚度范 围(3.0 5.0 mm)内其最小反射损耗均低于 $-20 \mathrm{~dB}$ (即 吸收率超过 $99 \%)$, 反射损耗小于 $-20 \mathrm{~dB}$ 的吸收频率 范围为 3.6 11.7 GHz，几乎覆盖整个 $\mathrm{C}$ 波段 $(4 \sim 8 \mathrm{GHz})$ 和 $\mathrm{X}$ 波段 $(8 \sim 12 \mathrm{GHz})$ 。当厚度为 $4.5 \mathrm{~mm}$ 时, 相应 涂层的吸波能力最强, 吸收峰位于 $6.6 \mathrm{GHz}$ 处, 与 其磁损耗峰基本吻合，此时最小反射损耗达到 $-56.9 \mathrm{~dB}$ 。从所得结果来看, NCZFO 纳米纤维的吸 波性能要明显优于同组分的 NCZFO 粉体 ${ }^{[27]}$, 后者 在吸收剂含量为 $85.7 \mathrm{wt} \%$ 、涂层厚度为 $3 \mathrm{~mm}$ 时, 最
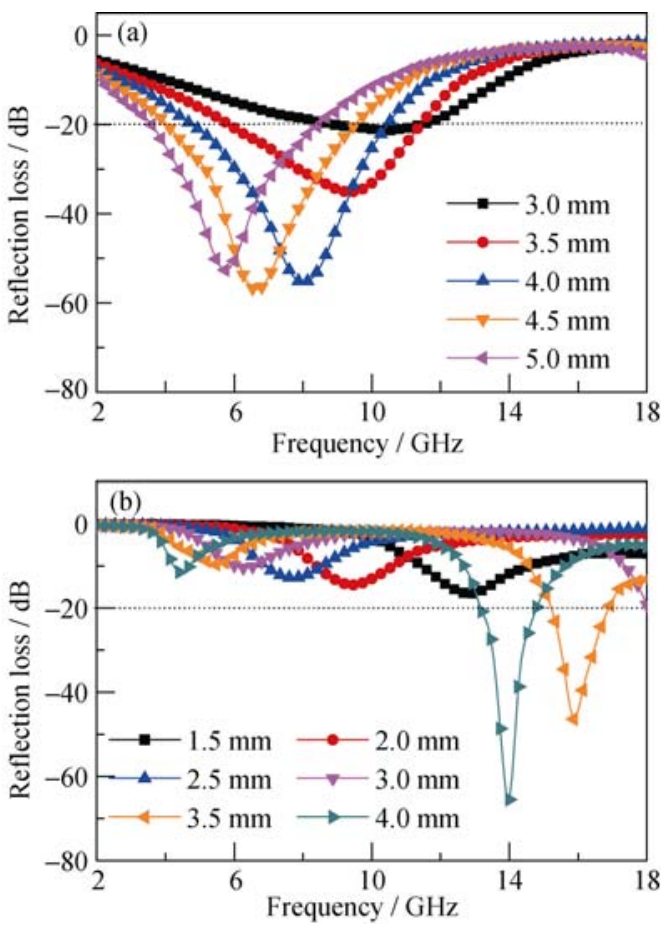

图 5 不同厚度 $\mathrm{S} 1(\mathrm{a})$ 和 $\mathrm{S} 2(\mathrm{~b})$ 单层吸波涂层的微波吸收曲线 Fig. 5 Microwave absorption curves of single-layer absorbers S1 (a) and S2 (b) absorbing coatings with different assumed thicknesses

小反射损耗仅达到 $-17.01 \mathrm{~dB}$ 。由图 5(b) 可见, $\mathrm{S} 2$ 单 层在厚度为 $1.5 \sim 4.0 \mathrm{~mm}$ 时, 反射损耗低于 $-20 \mathrm{~dB}$ 的 吸收带宽为 $4.9 \mathrm{GHz}$ ，频率范围为 $13.1 \sim 18 \mathrm{GHz}$ ，覆 盖了约 $82 \%$ 的 $\mathrm{Ku}$ 波段(12 18 GHz)，在厚度为 $4 \mathrm{~mm}$ 时反射损耗达到最小值约 $-65.5 \mathrm{~dB}$ 。由于 BTO 纳米 纤维在高频范围具有相对更大的电磁损耗, 因此 对电磁波的有效吸收也主要集中在高频区域，这 与文献报道的 BTO 纳米管 ${ }^{[18]}$ 和片状粉体 ${ }^{[20]}$ 的结果 相近，但其吸收强度要远高于后两者。BTO 纳米管 和片状粉体在填充量为 $70 \mathrm{wt} \%$ 的情况下，其最小 反射损耗仅分别为 -21.8 和 $-29.6 \mathrm{~dB}$ 。此外, 由图 5 还可观察到，随着厚度的增加, S1 和 S2 单层涂层 的吸收峰都逐渐向低频方向移动, 意味着它们的 吸收频率范围可通过改变涂层厚度进行简单有效 的调控; 对于 $\mathrm{S} 2$ 单层, 当厚度超过 $3 \mathrm{~mm}$ 时, 在高 频段还出现了第二个吸收峰。这些现象都是由于电 磁波在吸波涂层内发生尺寸共振造成的, 即当吸 波涂层厚度 $d$ 等于 $\lambda / 4$ ( $\lambda$ 为涂层中电磁波的波长) 的奇数倍时 ${ }^{[5,8]}$.

$$
d=\frac{n \lambda}{4}=\frac{n c}{4 f \sqrt{\left|\varepsilon_{\mathrm{r}}\right|\left|\mu_{\mathrm{r}}\right|}} \quad n=1,3,5, \cdots
$$

吸波涂层上下表面反射波的位相刚好相反而相互抵 消，产生较强的微波吸收，形成吸收峰。由公式(3) 
可知, 随涂层厚度增大, 吸收峰的峰值频率将逐渐 减小, 同时在所测的频率范围内可能出现更多的吸 收峰。

基于遗传算法和阻抗匹配原理，我们设计了以 $\mathrm{S} 1$ 为吸收层、S2 为匹配层的双层结构吸波涂层, 并 根据以下公式模拟评估了其微波吸收性能 ${ }^{[10,13]}$ :

$$
\begin{gathered}
Z_{\text {in }(2)}=\frac{Z_{0} \sqrt{\frac{\mu_{2 \mathrm{r}}}{\varepsilon_{2 \mathrm{r}}}}\left[Z_{\text {in }(1)}+Z_{0} \sqrt{\frac{\mu_{2 \mathrm{r}}}{\varepsilon_{2 \mathrm{r}}}} \tanh \left(j \frac{2 \pi f d_{2}}{c} \sqrt{\mu_{2 \mathrm{r}} \varepsilon_{2 \mathrm{r}}}\right)\right]}{Z_{0} \sqrt{\frac{\mu_{2 \mathrm{r}}}{\varepsilon_{2 \mathrm{r}}}}+Z_{\text {in }(1)} \tanh \left(j \frac{2 \pi f d_{2}}{c} \sqrt{\mu_{2 \mathrm{r}} \varepsilon_{2 \mathrm{r}}}\right)} \\
Z_{\text {in }(1)}=Z_{0} \sqrt{\mu_{1 \mathrm{r}} / \varepsilon_{1 \mathrm{r}}} \tanh \left[j\left(2 \pi f d_{1} / c\right) \sqrt{\mu_{1 \mathrm{r}} \varepsilon_{1 \mathrm{r}}}\right] \\
R L(\mathrm{~dB})=20 \lg \left|Z_{\text {in }(2)}-Z_{0} / Z_{\mathrm{in}(2)}+Z_{0}\right|
\end{gathered}
$$

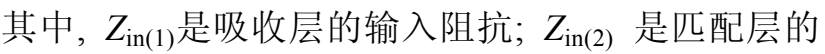
输入阻抗; $d_{1} 、 \varepsilon_{1 \mathrm{r}}$ 和 $\mu_{1 \mathrm{r}}$ 分别是吸收层的厚度、相对 复介电常数和相对复磁导率; $d_{2} 、 \varepsilon_{2 \mathrm{r}}$ 和 $\mu_{2 \mathrm{r}}$ 分别是吸 收层的厚度、相对复介电常数和相对复磁导率。图 6 给出了总厚度为 $3 \mathrm{~mm}$ 的双层吸波涂层的反射损 耗曲线。可以看出, 随吸收层 $(\mathrm{S} 1)$ 厚度从 $0.2 \mathrm{~mm}$ 增 加到 $2.9 \mathrm{~mm}$, 即匹配层厚度从 $2.8 \mathrm{~mm}$ 减少到 $0.1 \mathrm{~mm}$, 微波吸收峰向高频方向移动, 且峰值都低于 $-20 \mathrm{~dB}$, 反射损耗小于 $-20 \mathrm{~dB}$ 的频率范围为 4.9 12 $\mathrm{GHz}$ 。当 吸收层厚度为 $2.6 \mathrm{~mm}$ 、匹配层厚度为 $0.4 \mathrm{~mm}$ 时, 最 小反射损耗位于 $9.2 \mathrm{GHz}$ 达-84.5 dB。计算结果表 明, 与同厚度的单层吸波涂层相比, 所设计的双层 吸波涂层能够显著提高微波吸收性能。

为了优化双层吸收涂层的结构及性能, 我们进 一步考察了匹配层或吸收层厚度对微波吸收性能的 影响。图 7(a)给出了吸收层厚度固定为 $0.5 \mathrm{~mm}$, 匹配 层厚度为 $0.6 \sim 2.5 \mathrm{~mm}$ 的双层涂层的微波吸收曲线。 由图可见，随匹配层厚度由 $0.6 \mathrm{~mm}$ 提高到 $2.5 \mathrm{~mm}$,

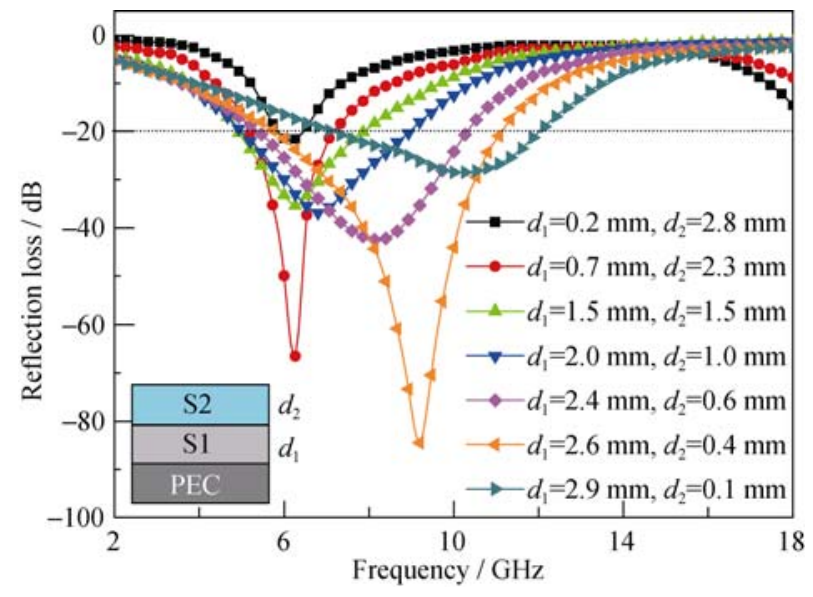

图 $63 \mathrm{~mm}$ 厚双层吸波涂层的微波吸收曲线

Fig. 6 Microwave absorption curves of $3 \mathrm{~mm}$ thick doublelayer absorbing coatings with $\mathrm{S} 1$ as absorbing layer and $\mathrm{S} 2$ as matching layer
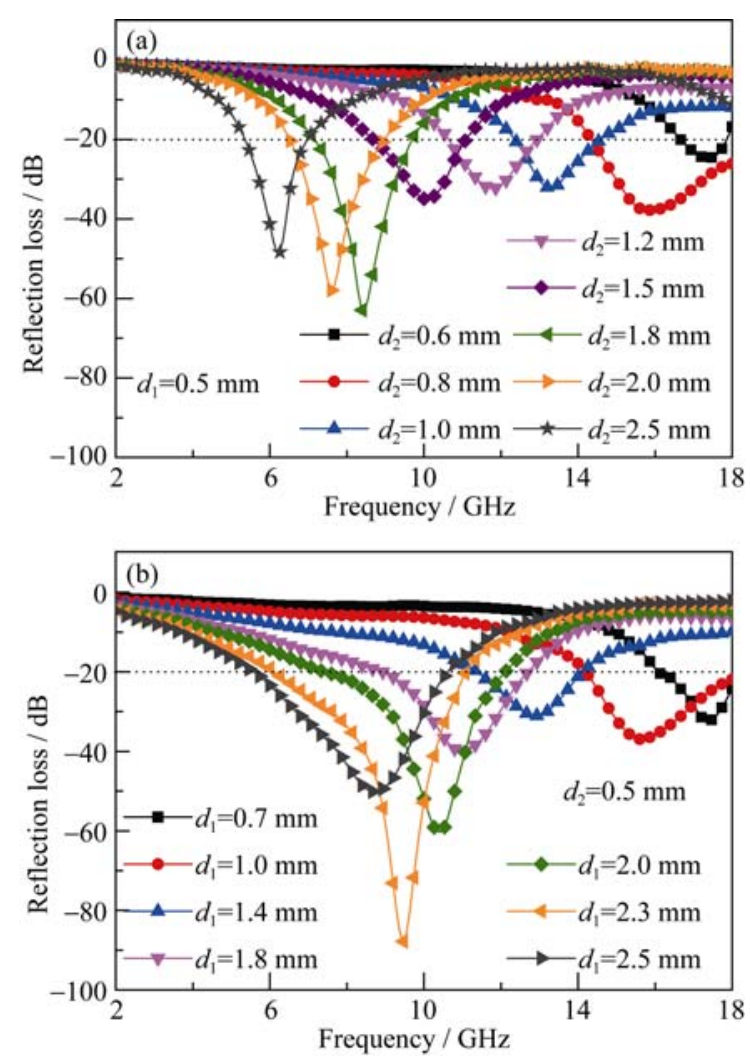

图 7 (a)吸收层厚为 $0.5 \mathrm{~mm}$ 、匹配层厚为 $0.6 \sim 2.5 \mathrm{~mm}$ 和(b) 匹配层厚为 $0.5 \mathrm{~mm}$ 、吸收层厚为 $0.7 \sim 2.5 \mathrm{~mm}$ 的双层吸波涂层 的反射损耗曲线

Fig. 7 Reflection loss curves of double-layer absorbing coatings (a) the thickness of the absorbing layer (S1) is $0.5 \mathrm{~mm}$ and the matching layer (S2) range from 0.6 to $2.5 \mathrm{~mm}$; (b) the thickness of the matching layer (S2) is $0.5 \mathrm{~mm}$ and the absorbing layer (S1) increases from 0.6 to $2.5 \mathrm{~mm}$

吸收峰逐渐移向低频区域, 吸收强度大体呈现先增 大后减小的变化趋势, 峰值均在 $-20 \mathrm{~dB}$ 以下, 小于 $-20 \mathrm{~dB}$ 的吸收带宽达 $12.6 \mathrm{GHz}$, 频率范围覆盖 $5.4 \sim 18 \mathrm{GHz}$ 。其中, 当匹配层厚度为 $1.8 \mathrm{~mm}$ 时, 吸 收峰峰值可达-63.1 dB。图7(b) 是匹配层厚度为 $0.5 \mathrm{~mm}$, 吸收层厚度为 $0.7 \sim 2.5 \mathrm{~mm}$ 的双层涂层的微波吸收 曲线。可以发现, 随吸收层厚度从 $0.7 \mathrm{~mm}$ 增加到 $2.5 \mathrm{~mm}$, 吸收峰也向低频方向移动, 微波吸收能力 也总体呈先增强后减弱态势, 低于 $-20 \mathrm{~dB}$ 的吸收频 率范围为 $5.5 \sim 18 \mathrm{GHz}$ 。在吸收层厚度为 $2.3 \mathrm{~mm}$ 时, 微波吸收达到最大值, 此时反射损耗为 $-87.8 \mathrm{~dB}$, 超过 $-20 \mathrm{~dB}$ 的吸收带宽为 $5 \mathrm{GHz}$, 频率范围为 6.1 11.1 GHz。结果反映出在保证良好吸收效果的 同时, 通过优化匹配层和吸收层可进一步降低整个 双层吸波涂层的厚度。

众所周知, 一个微波吸收材料的吸波性能主要 取决于其本身的电磁衰减能力以及与自由空间的阻 抗匹配特性。NCZFO 纳米纤维磁损耗与 BTO 纳米 
纤维介电损耗的结合能有效提高双层吸波涂层对电 磁波的衰减能力; 另外, 双层吸收涂层较单层的阻 抗匹配特性更好, 如图 8 所示, $3 \mathrm{~mm}$ 厚双层(匹配层 $0.4 \mathrm{~mm}$ 、吸收层 $2.6 \mathrm{~mm}$ )及 $\mathrm{S} 1$ 和 S2 单层的归一化 输入阻抗实部最大值分别为 $1.1 、 2.3$ 和 3.3 , 虚部最 大值分别为 $0.7 、 1.5$ 和 2.0 。与单层相比, 双层与自 由空间的归一化输入阻抗更接近(自由空间的实部 等于 1 , 虚部为 0$)$ 。因此, 磁损耗和介电损耗的结合 以及阻抗匹配特性的改善使双层结构具有更好的微 波吸收性能。

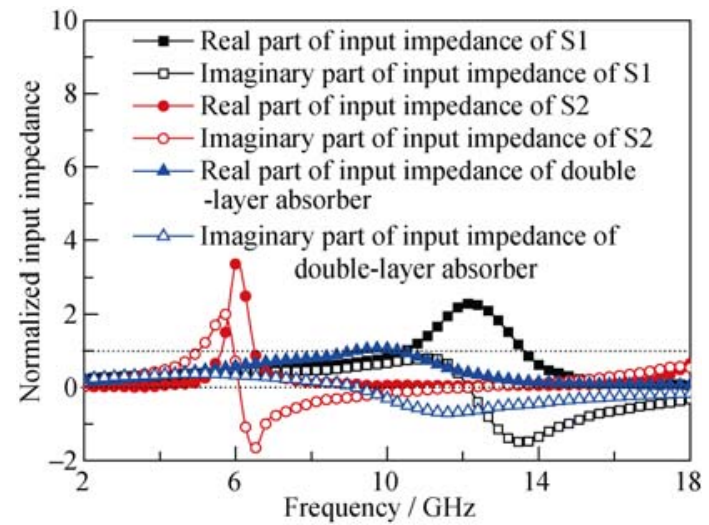

图 $83 \mathrm{~mm}$ 厚的单层与双层吸波涂层的归一化输入阻抗比较 Fig. 8 Comparison of the normalized input impedance between single-layer and double-layer absorbers with the same thickness of $3 \mathrm{~mm}$

\section{3 结论}

利用静电纺丝法结合热处理制备了结晶良好的 单相 NCZFO 纳米纤维和 BTO 纳米纤维, 模拟计算 和分析了以它们为吸收剂的硅橡胶基单层和双层结 构吸波涂层的吸收特性。与单层结构相比, 以 BTO 纳米纤维/硅橡胶复合物为匹配层、NCZFO 纳米纤 维/硅橡胶复合物为吸收层的双层结构不但具有更 好的微波吸收性能，而且还可有效降低整个涂层的 厚度。通过调控吸收层和匹配层的厚度，可使反射 损耗超过 $-20 \mathrm{~dB}$ 的吸收频率范围几乎覆盖整个 $\mathrm{C}$ 至 $\mathrm{Ku}$ 波段。当吸收层厚度为 $2.3 \mathrm{~mm}$ 、匹配层厚度为 $0.5 \mathrm{~mm}$ 时, 最小反射损耗达到 $-87.8 \mathrm{~dB}$, 低于 $-20 \mathrm{~dB}$ 的吸收带宽为 $5 \mathrm{GHz}$ ，频率范围为 $6.1 \sim 11.1 \mathrm{GHz}$ 。优 化设计的 NCZFO/BTO 纳米纤维双层结构复合吸波 材料在电磁波吸收与屏蔽领域具有良好的潜在应用 前景。

\section{参考文献:}

[1] CHE R C, PENG L M, DUAN X F, et al. Microwave absorption enhancement and complex permittivity and permeability of Fe encapsulated within carbon nanotubes. Adv. Mater, 2004, 16(5): 401-405.

[2] LI JIA, LIU HONG-BO, YANG LI. Research on microwave absorption properties of $\mathrm{FeCo} /$ graphite nanocomposite. Journal of Inorganic Materials, 2014, 29(5): 470-474.

[3] ZHANG X F, GUO J J, QIN G W. Assembled micro-nano particles with multiple interface polarizations for electromagnetic absorption at gigahertz. Appl. Phys. Lett., 2014, 104(25): 252404-1-4.

[4] SUN D P, ZOU Q, WANG Y P, et al. Controllable synthesis of porous $\mathrm{Fe}_{3} \mathrm{O}_{4} @ \mathrm{ZnO}$ sphere decorated graphene for extraordinary electromagnetic wave absorption. Nanoscale, 2014, 6(12): $6557-$ 6562.

[5] XIANG J, LI J, ZHANG X H, et al. Magnetic carbon nanofi bers containing uniformly dispersed $\mathrm{Fe} / \mathrm{Co} / \mathrm{Ni}$ nanoparticles as stable and high-performance electromagnetic wave absorbers. $J$ Mater. Chem. A, 2014, 2(40): 16905-16914.

[6] QING Y C, ZHOU, W C, LUO F, et al. Epoxy-silicone filled with multi-walled carbon nanotubes and carbonyl iron particles as a microwave absorber. Carbon, 2012, 48(14): 4074-4080.

[7] VINAYASREE S, SOLOMAN M A, SUNNY V, et al. A microwave absorber based on strontium ferrite-carbon black-nitrile rubble for S and X-band applications. Compos. Sci. Technol., 2013, 82: 69-75.

[8] MENG X G, WAN Y Z, LI Q Y, et al. The electrochemical preparation and microwave absorption properties of magnetic carbon fibers coated with $\mathrm{Fe}_{3} \mathrm{O}_{4}$ films. Appl. Surf. Sci., 2011, 257(24): 10808-10814.

[9] LIU Y, LIU X X, WANG X J. Double-layer microwave absorber based on $\mathrm{CoFe}_{2} \mathrm{O}_{4}$ ferrite and carbonyl iron composites. J. Alloys Compd., 2014, 584: 249-253.

[10] QING Y C, ZHOU W C, LUO F, et al. Optimization of electromagnetic matching of carbonyl iron $/ \mathrm{BaTiO}_{3}$ composites for microwave absorption. J. Magn. Magn. Mater, 2011, 323(5): 600-606.

[11] LIU Q L, CAO B, FENG C L, et al. High permittivity and microwave absorption of porous graphitic carbons encapsulating $\mathrm{Fe}$ nanoparticles. Compos. Sci. Technol., 2012, 72(13): 1632- 1636.

[12] XIE WEI, CHENG HAI-FENG, CHU ZENG-YONG, et al. Radar absorbing properties of light radar absorbing materials based on hollow porous carbon fibers. Journal of Inorganic Materials, 2009, 24(2): 320-324.

[13] XIANG JUN, ZHANG XIONG-HUI, YE QIN, et al. Structural design and absorption properties of double-layer microwave absorbers based on $\mathrm{Li}_{0.33} \mathrm{Zn}_{0.3} \mathrm{Fe}_{2.35} \mathrm{O}_{4}$ and carbon nanofibers. Chinese Journal of Inorganic Chemistry, 2014, 30(4): 845-852.

[14] HUANG X G, ZHANG J, XIAO S R, et al. The cobalt zinc spinel 
ferrite nanofiber: lightweight and efficient microwave absorber. $J$. Am. Ceram. Soc., 2014, 97(5): 1363-1366.

[15] ZHANG M, LIU C Q, ZI Z F, et al. Magnetic and microwave absorption properties of $\mathrm{Ni}_{1-x} \mathrm{Zn}_{x} \mathrm{Fe}_{2} \mathrm{O}_{4}$ nanocrystalline synthesized by Sol-Gel method. Sci. China Technol. Sci., 2013, 56(1): 13-19.

[16] Ma Z, Cao C T, Yuan J, et al. Enhanced microwave absorption of $\mathrm{BaTiO}_{3}$-based ferroelectric/ferromagnetic nanocomposite. Appl. Surf. Sci., 2012, 258(19): 7556-7561.

[17] XIE F, LIU J W, GU D, et al. Microwave absorption enhancement and electron microscopy characterization of $\mathrm{BaTiO}_{3}$ nano-torus. Nanoscale, 2011, 3(9): 3860-3867.

[18] ZHU Y F, ZHANG L, NATSUKI T, et al. Facile synthesis of $\mathrm{BaTiO}_{3}$ nanotubes and their microwave absorption properties. ACS Appl. Mater. Interfaces, 2012, 4(4): 2101-2106.

[19] YANG J, ZHANG J, LIANG C Y, et al. Ultrathin $\mathrm{BaTiO}_{3}$ nanowires with high aspect ratio: a simple one-step hydrothermal synthesis and their strong microwave absorption. ACS Appl. Mater. Interfaces, 2013, 3(15): 7146-7151.

[20] JING L N, WANG G Q, DUAN Y P, et al. Synthesis and electromagnetic characteristics of the flake-shaped barium titanate powder. J. Alloys Compd., 2009, 475(1/2): 862-868.
[21] GUAN P F, ZHANG X F, GUO J J. Assembled $\mathrm{Fe}_{3} \mathrm{O}_{4}$ nanoparticles on graphene for enhanced electromagnetic wave losses. Appl. Phys. Lett., 2012, 101(15): 153108-1-4.

[22] ZHANG Q, LI C F, CHEN Y N, et al. Effect of metal grain size on multiple microwave resonances of $\mathrm{Fe} / \mathrm{TiO}_{2}$ metal-semiconductor composite. Appl. Phys. Lett., 2010, 97(13): 133115-1-3.

[23] KONG L B, LI Z W, LIU L, et al. Recent progress in some composite materials and structures for specific electromagnetic applications. Int. Mater. Rev., 2013, 58(4): 203-259.

[24] MANDAL A, DAS C K. Effect of $\mathrm{BaTiO}_{3}$ on the microwave absorbing properties of Co-doped $\mathrm{Ni}-\mathrm{Zn}$ ferrite nanocomposites. $J$. Appl. Ploym. Sci., 2014, 131(4): 39926-1-9.

[25] LIU Q L, ZHANG D, FAN T X. Electromagnetic wave absorption properties of porous carbon/Co nanocomposites. Appl. Phys. Lett., 2008, 93(1): 013110-1-3.

[26] HUANG X, LU M, ZHANG X, et al. Carbon microtube/ $\mathrm{Fe}_{3} \mathrm{O}_{4}$ nanocomposite with improved wave-absorbing performance. Scripta Mater., 2012, 67(6): 613-616.

[27] ZHAO D L, LV Q, SHEN Z M. Fabrication and microwave absorbing properties of Ni-Zn spinel ferrites. J. Alloys Compd., 2009, 480(2): 634-638. 